\title{
Structure-Function Relations in E. coli 16S RNA
}

\author{
John F. Thompson* and John E. Hearst \\ Department of Chemistry \\ and the Laboratory of Chemical Biodynamics \\ University of California, Berkeley, \\ Berkeley, California 94720
}

\section{Introduction}

As a more thorough understanding of RNA secondary and tertiary structure has developed over the last several years, so has an appreciation of its importance in the function of the various RNP particles of which it is a part. For example, U1 RNA has been linked to mRNA splicing (Lerner et al., 1980; Rogers and Wall, 1980), while the RNA moiety of RNAase $P$ has been shown to be absolutely required for its activity (Kole et al., 1980). RNA has even been shown to be capable of making and breaking phosphodiester bonds in the complete absence of protein (Kruger et al., 1982). Along with these developments, the concept that ribosomal RNA is merely a framework on which ribosomal proteins can carry out their functions has been discarded. Indeed, speculations on the evolution of the protein-synthesizing system have generally concluded that the RNA must have predated the protein components. The similarity in structure of protein-free 16S RNA in solution and $16 \mathrm{~S}$ RNA in the 305 subunit (observed with psoralen crosslinking by Wollenzein et al., 1979; Thammana et al., 1979; Thompson and Hearst, 1983; and with electron microscopy) suggests that at least vestiges of the original catalytic structure remain. While Escherichia coli rRNA may no longer be able to carry out protein-free translation, it is now yenerally accepted that it plays an active role in ribosomal functions. Unfortunately, the dearth of structural information has allowed formulation of only simple models for how RNA operates.

Even though the sequence of 16S RNA is known (Brosius et al., 1978; Carbon et al., 1979) and much of its secondary structure is agreed on (Noller and Woese, 1981; Stiegler et al., 1981; Zwieb et al., 1981), little progress has been made towards linking specific structures with function. Recent work with psoralen crosslinking of 165 RNA (Thompson and Hearst, 1983) has confirmed parts of the secondary structure, and has also provided evidence for new interactions that appear to be functionally important. We will discuss how these structural features may be related to specific ribosomal mechanisms. We will concentrate on E. coli 16S RNA, but eucaryotic 18S RNA will also be presented when its function appears to be substantially different. Reference to most ribosomal proteins will be tastefully omitted, primarily because their interactions with the RNA are poorly understood, but also because we have approached the problem with the bias that they modulate the activity of the RNA rather than being the principal driving force behind it.

\footnotetext{
* Present address: Division of Biology and Medicine, Brown University,
} Providence, Rhode Island 02912.

\section{mRNA Binding}

The role of $16 S$ RNA in recognizing and binding mRNA in the initiation complex is well established (Shine and Dalgarno, 1975; Steitz and Jakes, 1975); but, in eucaryotes, the sequence that has been implicated in mRNA binding has been deleted. Because both eucaryotic and procaryotic small subunits perform essentially the same functions, there should be some compensating interaction between 18S RNA and mRNA. There are no apparent similarities in either the primary or secondary structure of eucaryotic mRNAs that would provide a basis for this.

The interaction 950-956/1507-1513, located by the psoralen crosslink GPs $956 \times 1506$ (for nomenclature see Thompson and Hearst, 1983), brings together two highly conserved regions in E. coli 16S RNA. In procaryotes and eucaryotes, there are a number of modified bases located in both these parts of the RNA. In E. coli, there are an $\mathrm{m}^{2} \mathrm{G}$ and an $m^{5} \mathrm{C}$ present in the region 550 bases from the $3^{\prime}$ end. In eucaryotes, these have been replaced by the hypermodified base am $\psi$ (Youvan and Hearst, 1981). $950-$ $956 / 1507-1513$ is conserved in eucaryotes, thus placing am $\psi$ spatially near what corresponds to the Shine-Dalgarno sequence of procaryotes, and suggesting that it may have a role in mRNA recognition. The modifications present on am $\psi$ allow it to make specific interactions with the $m^{7} G$ cap structure found at the $5^{\prime}$ end of all eucaryotic mRNAs. The negative charge delocalized on the carboxylic acid group of am $\psi$ can stabilize the positive charge delocalized on the imidizole nitrogens. Simultaneously, the amino group of am $\psi$ can interact with one of the negatively charged phosphate groups. Additional, indirect evidence for this interaction was presented by Thompson (1982). Efforts to obtain a more direct, experimental basis for the $a m \psi-m^{7} G$ interaction are now under way.

The equilibrium between 950-956/1507-1513 and 1506-1515/1520-1529 may also be involved in more complex intersubunit contacts. Azad (1979) has proposed an interaction between 5S RNA and the same region of 16S RNA (1509-1517) that pairs with the region near $\mathrm{m}^{2} \mathrm{G} \mathrm{m}^{5} \mathrm{C}$. There is no firm evidence for this interaction, and it was suggested by Schnare and Gray (1981) that it is not universal. However, stable base pairing of $5 S$ and $18 S$ RNA in solulion has been observed (Oakden et al., 1977). The in vitro complex formed between Drosophila melanogaster $18 \mathrm{~S}$ and $5 \mathrm{~S}$ RNAs can be crosslinked by hydroxymethyltrimethylpsoralen, and large amounts of $5 \mathrm{~S}$ copurify with D. melanogaster 18S RNA, even after two rounds of sucrose gradient centrifugation using standard purification protocols (Thompson, 1982). The fact that 305 subunits that contain EPs $956 \times 1506$ are less able to form 705 ribosomes than other crosslinked subunits (Thammana et al., 1979) further suggests that $5 S$ pairs with 165 through this interaction.

How these interactions might alternate through the ribosomal cycle is not clear. For instance, one interaction might only occur during initiation, while the others might 
switch during elongation. Psoralen crosslinking of ribosomes irradiated at specific points in translation will clarify this situation.

\section{Proofreading and tRNA Binding}

The total error rate in translation is simply a sum of the error rates of its component reactions. The theoretical and practical problems involved in the analysis of translational fidelity are reviewed by Kurland (1980) and Yarus (1979). Best estimates place the total error rate from all factors at one misincorporation per $10^{4}$ amino acids. The only step in translation that cannot be expected to yield this level of discrimination easily is tRNA binding via the codon-anticodon interaction. The difference in binding energies of two tRNAs that contain partially degenerate anticodons for a single codon is far too small to expect such accurate reading. To account for this, a number of models have been presented, all of which involve reading the anticodon twice to multiply small differences in binding. The lack of experimental data has, up until now, prevented formulation of a detailed physical model of this process that satisfactorily accounts for what little is known.

No part of 16S RNA has been associated with a proofreading function. Several proteins, however, are known to be involved in regulating translational fidelity. Elongation factor Tu and S4, S11, S12, and S17 have all been shown to affect the error rate profoundly (Gavrilova et al., 1981, and references therein). The characteristics of one of the long-range crosslinks observed, GPs $625 \times 1420$, suggest that it might have a role in proofreading and tRNA binding. The region near 1420 has been implicated in binding of tRNA to the $P$ site (Taylor et al., 1981), and the region near 625 is part of the S8-binding site. S8, while not necessarily binding tRNA directly, has an effect on the amount bound (Nomura et al., 1969). Both regions are highly variable, as would be expected for a proofreading function. The proofreading process requires energy, and each species will have different requirements for optimizing the advantages of increased accuracy with the disadvantages of energy loss. Thus the details of proofreading should be different, even among closely related species, with larger differences upon going from mitochondria to procaryotes to eucaryotes. The region of $E$. coli $16 S$ RNA around $580-660$ varies considerably among procaryotes, and has been deleted entirely by mitochondria. Virtually all of the nucleotides that have been inserted into eucaryotic 18S RNA are found in this region. A similar behavior is seen around 1420. Mitochondria hàve shortened that helical stem, while eucaryotes have expanded it.

Intuitively, one might expect eucaryotes to require the lowest error rate and hence to devote more of the $18 \mathrm{~S}$ RNA to translational fidelity. Eucaryotes synthesize many more proteins than procaryotes and are thus more sensitive to error-induced damage. In contrast, mitochondria are almost free of proofreading constraints. All proteins synthesized are present as multiple copies, and only a few different ones are made. Indeed, all proteins that could propagate errors-ribosomal proteins (with a couple of exceptions) and polymerases - are synthesized outside the mitochondria. In some cases, mitochondria only read two of the anticodon nucleotides (Heckman et al., 1980). so that it would not be surprising if they were to delete all or part of the proofreading apparatus. We are unable to rely on experimental results because measurements of in vivo translational fidelity are extremely difficult to do and have been restricted to studies that detect only one or a few different misincorporated amino acids in a protein (Edelmann and Gallant, 1977). Even these studies have to be viewed critically, because E. coll ribosomes can reject nascent peptides that contain an error (Caplan and Menninger, 1979). Thesc oligopeptides are broken down rapidly in the cell, and thus are difficult to quantitate.

Lake (1979) has proposed a detailed model for what he terms the $R$ (recognition) site of tRNA binding. The anticodon is read once in the $R$ site. A conformational change in the tRNA occurs to bring it to the $A$ site, where the anticodon is read again. A primary reason for placing the $R$ site on the exterior of the 30 S subunit is the location of several tRNA-binding and proofreading proteins there (including S8). For the reasons mentioned above, the cyclic interaction of $612-617 / 623-628 \rightleftharpoons 620-626 / 1420-1426$ appears to be ideally suited for involvement in the process of moving a tRNA from the R site to the A site.

Lake (1981) proposes that the conformational change that brings the tRNA to the $A$ site would occur solely in the tRNA, with the only contact to the ribosomal complex being at the anticodon. This seems unlikely not only because of the weakness of some codon-anticodon interactions, but also because of the ease with which the process could be short-circuited. If the IRNA in the process of switching were to come off the mRNA, there would be nothing to prevent a new tRNA that had not undergone the initial screening at the $R$ site from taking its place and moving into the $A$ site. It is more likely that there are multiple tRNA-protein and tRNA-rRNA contact points that ensure that the bound tRNA has all the imporlant fealures of the cognate aminoacyl-tRNA. In this way, other conformational changes in the ribosome could be tightly coupled to tRNA movement.

Elongation factor Tu has been shown to recognize the $3^{\prime}$ end of aminoacyl-tRNA before binding to the ribosome (reviewed by Weissbach, 1980). The tRNA-binding proteins on the exterior of the $30 \mathrm{~S}$ subunit and elongation factor Tu make the contacts with the tRNA in the R site. It would not be surprising if rRNA were also involved. The high variability of the 588-617/623-651 region suggests that it is not directly involved in tRNA binding. There are, however, two nearby sequences of CGAA that are highly conserved. Both of these stretches, located at 726-729 and 764-767 in E. coli, are present in all procaryotes and eucaryotes, and at least one is present in all mitochondria. CGAA is complementary to the highly conserved T $\psi \mathrm{CG}$ present in tRNA. While this sequence is not available for 
inter-RNA binding in solution, there is strong evidence that binding of a codon to tRNA makes this region more accessible (reviewed by Kim, 1978) and thus able to bind to 16S RNA or 5S RNA. Such an interaction would destroy contact between the $\mathrm{D}$ and $\mathrm{T} \psi$ loops of tRNA, also freeing other sequences for interaction. The conserved YGG sequence in the $\mathrm{D}$ loop could also be involved in interactions in the $\mathrm{R}$ site, or it might remain free to allow specific binding to the A site upon switching.

There is a strongly conserved sequence in 16S RNA that would allow pairing of the $Y G G$ sequence in the $A$ site and subsequently in the $P$ site. The sequence CCGm ${ }^{4} \mathrm{CmCCG}(1399-1405$ in E. coli) is present in all procaryotes and eucaryotes and some mitochondria. In the A site, 1399-1401 would pair wilt the exposed YGG, while the P-site tRNA YGG could pair with 1403-1405.

This hypothesis is based partly on the data of Ofengand et al. (1982). They found that a modified base in the anticodon loop of a P-site tRNA could crosslink to C-1400. This crosslinking was done with an empty $A$ site, so C1400 would be available. When the proper codon was supplied for the tRNA (Ofengand and Liou, 1981), crosslinking was abolished. This indicates that the interaction between the anticodon and C-1400 is probably not functionally important. It does, however, establish that the $\mathrm{P}$ site is in very close proximity. The A site must also be very close, because Johnson et al. (1982) found that the distance between the $\mathrm{S}^{4} \mathrm{U}$ position in tRNAs bound to the $A$ and $\mathrm{P}$ sites is only $2-10 \AA$ greater than the tRNA diameter. This implies that the A- and P-site tRNAs are in very close contact throughout their entire lengths, because the anticodon loops and $3^{\prime}$ ends also have to be quite close.

For the D loops to pair with 16S RNA as described, the tRNA would have to undergo a conformational change. The $\mathrm{D}$ and $\mathrm{T} \psi$ loops would have already separated in the $R$ site. The $D$ loop and stem would also have to twist slightly and move toward the anticodon loop. In such a conformation, the bases in the anticodon and D loops are in an antiparallel configuration. The $A$ site is on the $3^{\prime}$ side of the mRNA; thus it is on the 5' side of the 16S RNA. A diagram of how this might happen is shown in Figure 1. The mRNA is necessarily kinked so the anticodon loops can base-pair to adjacent codons on the mRNA. There is a larger distance allowable between the $D$ loops because the base-paired regions are separated by an unpaired, modified nucleotide. The base and sugar methylations might somehow stabilize this kink.

Except for a few mitochondrial tRNAs and tRNAs involved in cell-wall synthesis, the sequence GG is present in the same location in the $D$ loop. The base $5^{\prime}$ to this is usually a $C, D$, or $U$, but occasionally an $A$. The pairing of G-1401 or G-1405 with this variable base holds additional potential for distinguishing between different tRNAs. The structures of GC, GU, GD, and GA pairs are all different and may change the orientation of a tRNA enough that, depending on the remainder of the structure, it could stabilize cognate and destabilize noncognate tRNAs.

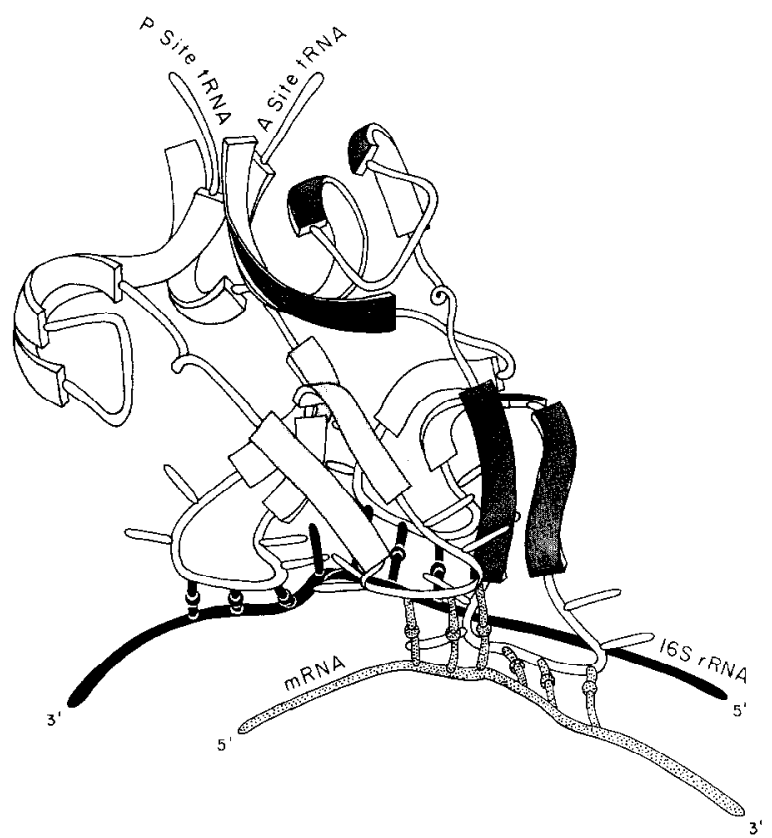

Figure 1. Possible Structure of Two tRNAs Interacting with Both mRNA and 165 RNA in the $A$ and $P$ Sites of the Ribosome

\section{Translocation and Elongation}

Very little is known about the mechanism of translocation and which parts of the ribosome are involved. The following model for translocation and movement of tRNAs through the ribosome is presented not as a definitive statement on how the ribosome works, but as a way of accounting for our observations and those of other workers in the field. There is clearly much work to be done, and this model should help point out weaknesses in our knowledge.

The tRNAs appear to have an essential role in the mechanism of translocation. The distance that mRNA moves is determined by the tRNA (Thach and Thach, 1971; Gupta et al., 1971). Johnson et al. (1982) have also proposed that the energy for translocation comes from energy stored when the A-site tRNA is tightly packed adjacent to the P-site tRNA. For the tRNAs to be in such close contact, there must be other parts of the ribosome that prevent the tRNAs from escaping. Since this would necessarily have to be a cyclic interaction, RNA-RNA interactions seem likely to be involved. While intersubunit interactions could play a role in this, we have no information that relates to this. A large part of the tRNA-binding sites are localized on the 30 S subunits, so 16S RNA could certainly play a major role.

Brimacombe (1980), while not setting forth a specific model, proposcd that $39 \cdot 47 / 393-402+1055-1065 /$ 1186-1195 $\rightleftharpoons 385-399 / 1052-1067$ might somehow be involved in translocation. There is no direct evidence for Brimacombe's proposal, but this seems to be exactly the type of cyclic, long-range interaction necessary if translocation is to proceed as described above. The interactions shown in Figure 2 are certainly intricate enough to lock the 


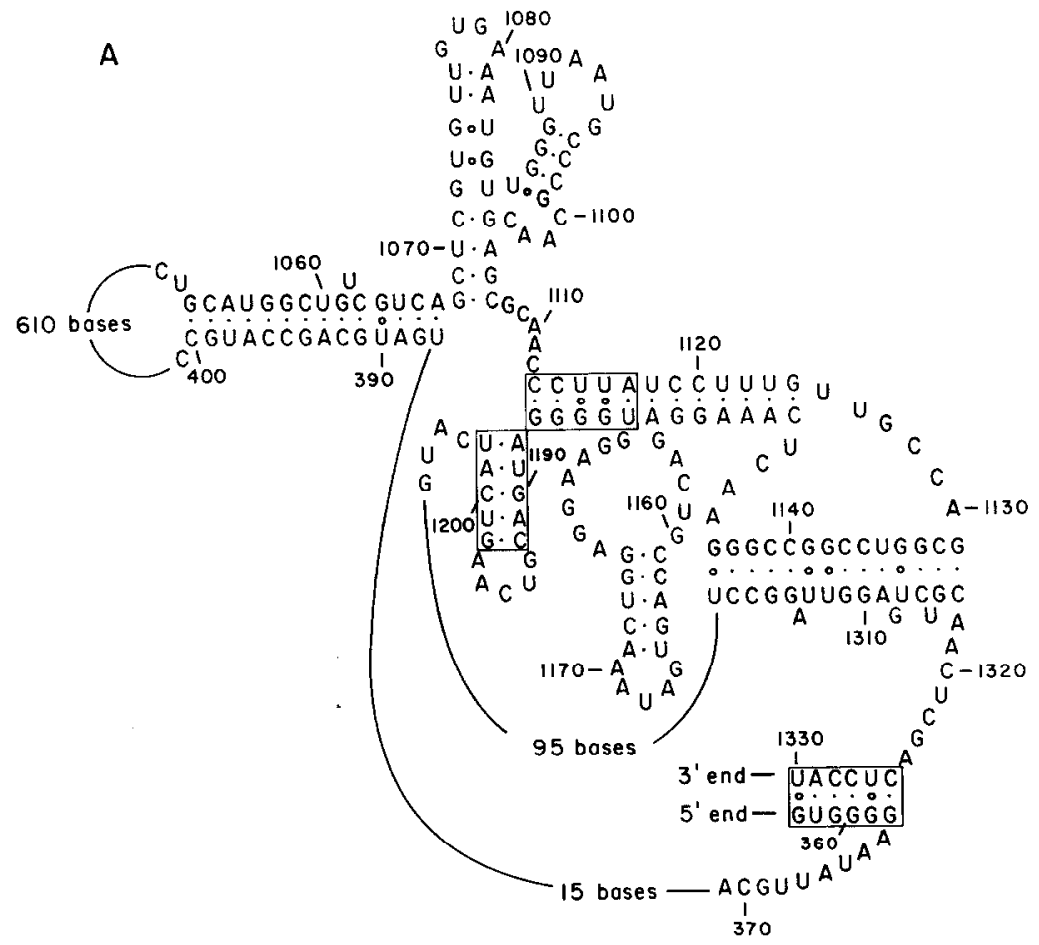

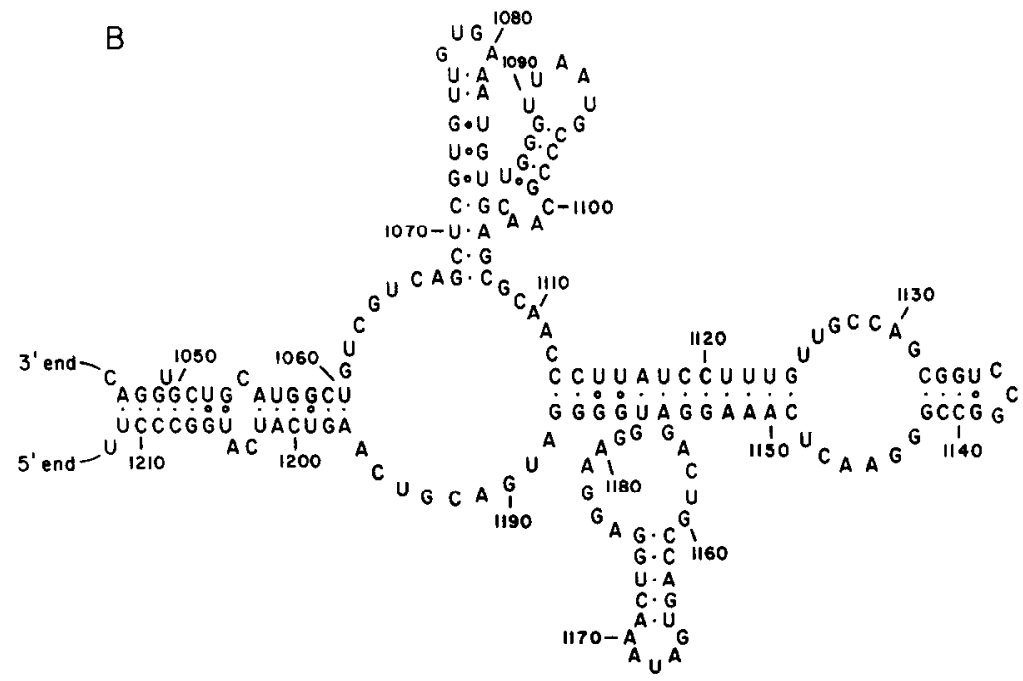

Figure 2. Possible Conformational Changes in 16 RNA

(A) Long-range interactions shown are supported by three different psoralen crosslinks (boxed) and one interaction found by Glotz et al. (1981), 387400/1053-1067. 1131-1144/1301-1317 is not present in any of the current models, but similar structures can be drawn for other species.

(B) Short-range interactions shown are from the newest version of the Noller and Woese (1981) model (H. Noller, personal communication).
$P$ site tRNA in place. At the opposite end of the $30 \mathrm{~S}$ subunit, similar interactions would have to occur to lock the A-site tRNA in place. These could include 950-956/ 1507-1513 and other interactions such as EPs $450 \times$ 1540, EPs $510 \times 1540$, or EPs $0 \times 1540$, which have been mapped by electron microscopy (Wollenzein et al., 1979; Wollenzein and Cantor, 1982), but which are not known to a sufficiently high degree of resolution to describe in detail.

A chart showing our proposed model for the elongation process is shown in Figure 3 . The role of elongation factors and conformational changes is shown, with the occupancy of the tRNA-binding sites after each event listed.

When elongation factor G-GTP binds to the pretranslocation ribosome, it destabilizes the long-range interactions and causes the short-range base pairing shown in Figure $2 \mathrm{~B}$ to occur. This provides an escape route for the P-site tRNA. This tRNA is rapidly expelled from the $P$ site because of electrostatic repulsion from the A-site tRNA. The A-site tRNA moves to the $P$ site simultaneously because of the much greater affinity of peptidyl-tRNAs for the $P$ site. Atter tRNA movement, elongation factor G-GDP dissociates from the ribosome, catalyzed by GTP hydrolysis. The stabilization by elongation factor $G$ of short-range interactions is no longer a factor in determining which conformation is favored; thus the interdomain interactions shown in Figure $2 \mathrm{~A}$ are re-established.

Evidence for an additional binding site for deacylated tRNA was found by Wettstein and Noll (1965). Additional support for this site, as well as a functional rationale for it, 


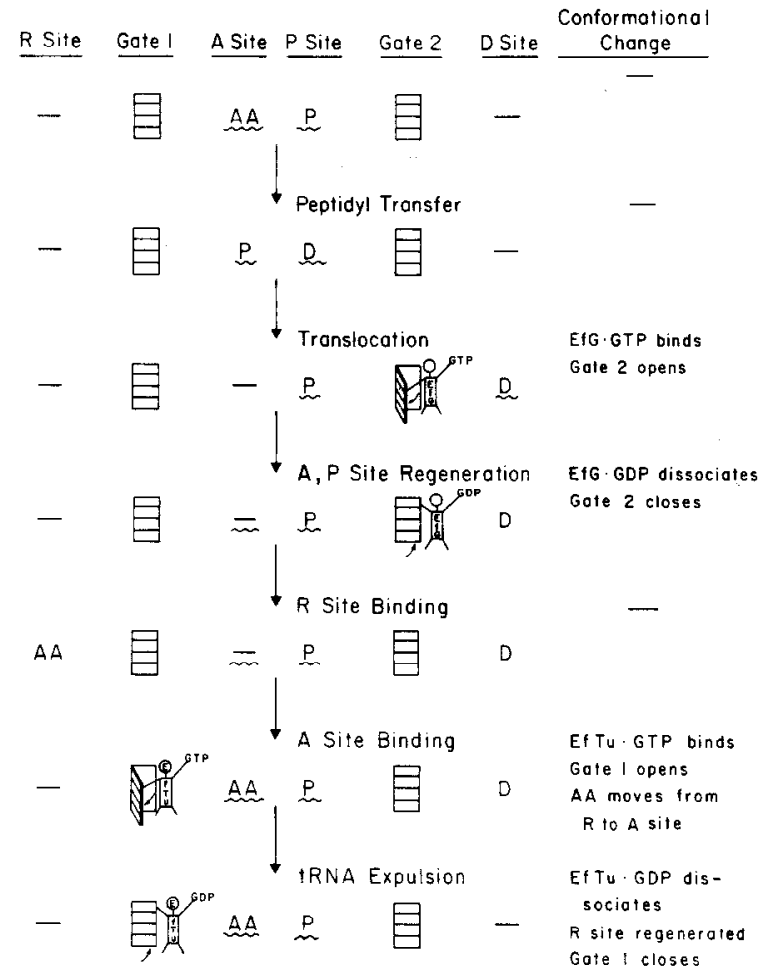

Figure 3. Model for Elongation and Translocation

The model involves six steps, three of which involve structural transitions in the RNA. These transitions are described below.

First, gate 1 is a steric barrier to tRNA movement at one end of the cleft in the $30 S$ subunit. It separates the $R$ and $A$ sites, and is associated with the following, and possibly other, transitions in secondary structure:

gate 1 (closed) $\leftrightarrow$ gate 1 (open)

950-956/1507-1513 $\leftrightarrow 946-955 / 1225-1235+1506-1515 / 1520-1529$

Second, gate 2 is a similar barrier at the other end of the cleft between the $\mathrm{P}$ and $\mathrm{D}$ sites. It is associated with the transition described in Figure 2.

gate 2 (closed) $\leftrightarrow$ gate 2 (open)

Figure $2 \mathrm{~A} \leftrightarrow$ Figure $2 \mathrm{~B}$

Third, the movement of the aminoacyl-tRNA from the $R$ site to the A site is associated with the following transition:

aminoacyl-tRNA (R site) $\leftrightarrow$ aminoacyl-tRNA (A site)

$612-617 / 623-628 \leftrightarrow 620-626 / 1420-1426$

During the next step (tRNA expulsion), the reversal of this structural change in the 165 RNA occurs, leaving the aminoacyl-tRNA in the A site and generating an empty $R$ site.

In the figure, four sites of IRNA binding are postulated. The $\mathrm{R}$ (recognition) site corresponds, in principle, to that described by Lake (1981). The physical attributes and position are not necessarly the same, however. The $A$ and $P$ sites are as usually proposed. The $D$ (discharge) site corresponds to the $E$ site of Rheinberger et al. (1981). The name has been changed for acronymic reasons. AA: aminoacyl-tRNA. D: deacylated tRNA. Wavy lines: positions of the CCG sequences in the 16S RNA, which base-pair with the $\mathrm{D}$ loop of the TRNA (see text). Ef: elongation factor.

was provided by Nierhaus et al. (1980) and Rheinberger et al. (1981) on the basis of filter-binding studies. Velocity sedimentation, however, has yielded ambiguous results on this point (Schmitt et al., 1982; Grajevskaja et al., 1982). Whether this is caused simply by differences in ribosomal preparations or by more serious inconsistencies is not clear. In any case, the D site (the $E$ site has been renamed the $\mathrm{D}$ site for acronymic reasons) has been included in our model because, at present, the weight of evidence suggests that it is real. The magnitude of the dissociation constant may be strongly dependent on the method of ribosome preparation, but even a high dissociation rate might enhance accuracy in vivo. The presence of the deacylated tRNA in the $D$ site accelerates the binding of the aminoacyl-tRNA-elongation factor Tu-GTP ternary complex to the ribosome. This occurs after an initial reading of the anticodon of the incoming tRNA. Once the anticodon has been interpreted as correct, elongation factor Tu-GTP binds with high affinity to the short-range interactions near the A site (946-955/1225-1235 + 1506-1515/1520$1529)$ and allows the R-site tRNA to move into the A site. In the presence of elongation factor $\mathrm{Tu}$, this movement is irreversible and provides the nonequilibrium situation necessary for true proofreading to occur (Yarus, 1979; Kurland, 1980). Once in place, the tRNA anticodon is reread. If still deemed correct, elongation factor Tu-GDP dissociates from the ribosome with hydrolysis of GTP. This allows the long-range interactions to re-form (950-956/ 1507-1513) and locks the two tRNAs in place, correctly positioned for peptidyl transter.

This model is necessarily incomplete, but does account for all the data available on elongation at present. For instance, while four tRNA-binding sites are proposed, only two or three are occupied at any one time. This agrees with the data of Rheinberger et al. (1981), who found 22.5 tRNAs bound during translation. It also includes the $R$ and $D$ sites, which increase the fidelity of translation (Lake, 1981; Nierhaus et al., 1980). The properties of noncleavable GIP analogs in factor binding can be accounted for, because the energy input is used solely to favor one direction in a conformational equilibrium. Nonenzymatic translation is possible because the same conformational equilibrium would be present in the absence of factors but would simply occur at a slower rate. In this case, the only energy input would be from peptide-bond formation.

\section{Acknowledgments}

This work was supported by the Office of Energy Research, Office of Health and Environmental Research, Health Effects Research Division, of the U.S. Department of Energy under contract number DE-AC0376SF00098, and by a grant (GM-11180) from the National Institutes of Health.

Received October 18, 1982; revised January 21, 1983

\section{References}

Azad, A. A. (1979). Intermolecular base-paired interaction between complementary sequences present near the $3^{\prime}$ end of 5S rRNA and 18S (16S) IRNA might be involved in the reversible association of ribosomal subunits. Nucl. Acids Res. 7, 1913-1929.

Brimacombe, R. (1980). The experimental determination of RNA structure and $\mathrm{RN} \wedge$-protein neighbourhoods in $E$. coli ribosomal subunits, in Biologica Implications of Protein-Nucleic Acid Interactions, J. Augustyniak, ed. (Amsterdam: Elsevier/North-Holland Biomedical Press), pp. 44-62.

Brosius, J., Paimer, M. L., Kennedy, P. J., and Noller, H. F. (1978). Complete nucleotide sequence of a 165 ribosomal RNA gene from Escherichia coli. Proc. Nat. Acad. Sci. USA 75, 4801-4805.

Caplan, A. B., and Menninger, J. R. (1979). Tests of the ribosomal editing 
hypothesis: amino acid starvation differentially enhances the dissociation of peptidyl-tRNA from the ribosome. J. Mol. Biol. 134, 621-637.

Carbon, P., Ehresmann, C., Ehresmann, B., and Ebel, J.P. (1979). The complete nucleotide sequence of the ribosomal 16S RNA from Escherichia coli. Eur. J. Biochem. 100, 399-410.

Edelmann, P., and Gallant, J. (1977). Mistransiation in E. coli. Cell 10, 131137.

Gavrilova, L. P., Perminova, I. N., and Spirin, A. S. (1981). Elongation factor Tu can reduce translation errors in poly $(U)$-directed cell-free systems. J. Mol. Biol. 149, 69-78.

Glotz, C., Zwieb, C., Brimacombe, R., Edwards, K., and Kössel, H. (1981). Secondary structure of the large subunit ribosomal RNA from Escherichia coli, Zea mays chloroplast, and human and mouse mitochondrial ribosomes. Nucl. Acids Res. 9, 3287-3306.

Grajevskaja, R. A., Ivanov, Y. V., and Saminsky, E. M. (1982). 70 r ribosomes of Escherichia coli have an additional site for deacylated IRNA binding Fur. J. Biochem. 128, 47-52

Gupta, S. L., Waterson, J., Sopori, M. L., Weissman, S. M., and Lengyel, $P$. (1971). Movement of the ribosome along the messenger ribonucleic acid during protein synthesis. Biochemistry 10, 4410-4421.

Heckman, J. E., Sarnoff, J, Alzner-DeWeerd, B., Yin, S., and RajBhandary, U. L. (1980). Novel features in the genetic code and codon reading patterns in Neurospora crassa mitochondria based on sequences of six mitochondrial tRNAs. Proc. Nat. Acad. Sci. USA 77, 3159-3163.

Hickey, E. D., Weber, L. A., Baglioni, C., Kim, C. H., and Sarma, R. H. (1977). A relation between inhibition of protein synthesis and conformation of 5'-phosphorylated 7-methylguanosine derivatives. J. Moł. Biol. 109, 173183.

Johnson, A. E., Adkins, H. J., Matthews, E. A., and Cantor, C. R. (1982). Distance moved by transfer RNA during translocation from the $A$ site to the P site on the ribosome. J. Mol. Biol. 156, 113-140.

Kim, S.H. (1978). Crystal structure of yeast tRNA ${ }^{\text {Phe, }}$; its correlation to the solution structure and functional implications. In Transfer RNA, S. Altman, ed. (Cambridge, Mass.: MIT Press), pp. 248-293.

Kole, R., Baer, M. F., Stark, B. C., and Altman, S. (1980). E. coli RNAase P has a required RNA component in vivo. Cell 19, 881-887.

Kruger, K., Grabowski, P. J., Zaug, A. J., Sands, J., Gottschling, D. E., and Cech, T. R. (1982). Self-splicing RNA: autoexcision and autocyclization of the ribosomal RNA intervening sequence of Tetrahymena. Cell 31, 147157

Kurland, C. G. (1980). On the accuracy of elongation. In Ribosomes, G. Chambliss et al., eds. (Baltimore: University Park Press), pp. 597-614.

Lake, J. A. (1979). Kibosome structure and tRNA binding sites. In I ransfer RNA: Structure, Properties, and Recognition, P. R. Schimmel et al., eds. (Cold Spring Harbor, New York: Cold Spring Harbor Laboratory), pp. 393411.

Lake, J. A. (1981). The ribosome. Sci. Am. 245, 84-97.

Lerner, M. R., Boyle, J. A., Mount, S. M., Wolin, S. L., and Steitz, J. A. (1980). Are snRNPs involved in splicing? Nature 283, 220-224.

Nierhaus, K. H., Rheinberger, H. J., Wurmbach, P., and Bergemann, K. (1980). Thermodynamic parameters of ribosomal tRNA binding and the importance of codon-anticodon interaction at the ribosomal P-site. In Riological Implications of Protein-Nucleic Acid Interactions, J. Augustyniak, ed. (Amsterdam: Elsevier/North-Holland Biomedical Press), pp. 121-131.

Noller, H. F., and Woese, C. R. (1981). Secondary structure of 165 ribosomal RNA. Science 212, 403-411.

Nomura, M., Mizushima, S., Ozaki, M., Traub, P., and Lowry, C. V. (1969). Structure and function of ribosomes and their molecular components. Cold Spring Harbor Symp. Quant. Biol. 34, 49-61.

Oakden, K. M., Azad, A. A., and Lane, B. G. (1977). Wheat embryo ribonucleates. VII. Rapid, efficient and selective formation of $5 S-18 S$ and $5.85-265$ hybrids in an aqueous solution of the four ribosomal polynucleotides, and the results of a search for the corresponding hybrids in wheat embryo ribosomes. Can. J. Biochem. 55, 99-109.

Ofengand, J., and Liou, R. (1981). Correct codon-anticodon base pairing at the $5^{\prime}$-anticodon position blocks covalent cross-linking between transfer ribonucleic acid and $16 \mathrm{~S}$ RNA at the ribosomal P site. Biochemistry 20 , 552-559.

Ofengand, J., Gornicki, P., Chakraburtty, K., and Nurse, K. (1982). Functional conservation near the $3^{\prime}$ end of eukaryotic small subunit RNA: photochemical crosslinking of $P$ site-bound acetylvalyl-tRNA to 18 S RNA of yeast ribosomes. Proc. Nat. Acad. Sci. USA 79, 2817-2821.

Rheinberger, H.-J., Sternbach, H., and Nierhaus, K. H. (1981). Three tRNA binding sites on Escherichia colf ribosomes. Proc. Nat. Acad. Sci. USA 78, 5310-5314.

Rogers, J., and Wall, R. (1980). A mechanism for RNA splicing. Proc. Nat. Acad. Sci. USA 77, 1877-1879.

Schmitt, M., Neugebauer, U., Bergmann, C., Gassen, H. G., and Riesner, D. (1982). Binding of tRNA in different functional states to Escherichia coli ribosomes as measured by velocity sedimentation. Eur. J. Biochem. 127, 525-529.

Schnare, M. N., and Gray, M. W. (1981). 3'terminal nucleotide sequence of Crithidia fasciculata smail ribosomal subunit RNA. FEBS Lett. 128, 298304.

Shine, J., and Dalgarno, L. (1975). Determinant of cistron specificity in bacterial ribosomes. Nature 254, 34-38.

Steitz, J. A., and Jakes, K. (1975). How ribosomes select initiator regions in MRNA: base pair formation between the $3^{\prime}$ terminus of 16S rRNA and the MRNA during initiation of protein synthesis in Escherichia coli. Proc. Nat. Acad. Sci. USA 72, 4734-4738.

Stiegler, P., Carbon, P., Ebel, J.-P., and Ehresmann, C. (1981). A general secondary structure model for procaryotic and eucaryotic RNAs of the small ribosomal subunits. Eur. J. Biochem. 120, 487-495.

Taylor, B. H., Prince, J. B., Ofengand, J., and Zimmerman, R. A. (1981). Nonanucleotide sequence from $16 \mathrm{~S}$ ribonucleic acid at the peptidyl transfer ribonucleic acid binding site of the Escherichia coli ribosome. Biochemistry 20, 7581-7588.

Thach, S. S., and Thach, R. E. (1971). Translocation of messenger RNA and "accommodation" of PMet-tRNA. Proc. Nat. Acad. Sci. USA 68, 17911795.

Thammana, P., Cantor, C. R., Wollenzcin, P. L., and Hearst, J. E. (1979). Crossinking studies on the organization of the $16 S$ ribosomal RNA within the 30 S Escherichia coli ribosomal subunit. J. Mol. Biol. 135, 271-283.

Thompson, J. F. (1982). Reaction of psoralen with RNA: specificity and use as a probe for secondary structure analysis. Ph.D. thesis, University of California, Berkeley, California.

Thompson, J. F., and Hearst, J. E. (1983). Structure of E. coli $16 \mathrm{~S}$ RNA elucidated by psoralen crosslinking. Cell 32, 1355-1365.

Vasiliev, V. D., Selivanov, O. M., and Kotelliansky, V. F. (1978). Specific self packing of the ribosomal 16 S RNA. FEBS Lett. 95, 273-276.

Weissbach, H. (1980). Soluble factors in protein synthesis. In Ribosomes, G. Chambliss et al, ed. (Baltimore: University Park Press), pp. 377-411.

Wettstein, F. O., and Noll, H. (1965). Binding of transfer ribonucleic acid to ribosomes engaged in protein synthesis: number and binding properties of ribosomal binding sites. J. Mol. Biol. 11, 35-53.

Wollenzein, P. L., and Cantor, C. R. (1982). Gel electrophoretic technique for separating crosslinked RNAs. Application to improved electron microscopic analysis of psoralen crosslinked $16 \mathrm{~S}$ ribosomal RNA. J. Mol. Biol. 159, 151-166.

Wollenzein, P. L., Hearst, J. E., Thammana, P., and Cantor, C. R. (1979). Base-pairing between distant regions of the Escherichia coli $16 \mathrm{~S}$ ribosomal RNA in solution. J. Mol. Biol. 135, 255-269.

Yarus, M. (1979). The accuracy of translation. Prog. Nucl. Acid Res. Mol. Biol. 23, 195-225.

Youvan, D. C., and Hearst, J. E. (1981). A sequence from Drosophila melanogaster 18S rRNA bearing the conserved hypermodified nucleoside amw: analysis by reverse transcription and high-performance liquid chromatography. Nucl. Acids Res. 9, 1723-1741.

Zwieb, C., Glotz, C., and Brimacombe, R. (1981). Secondary structure comparisons between small subunit ribosomal RNA molecules from six different species. Nucl. Acids Res. 9, 3621-3640. 